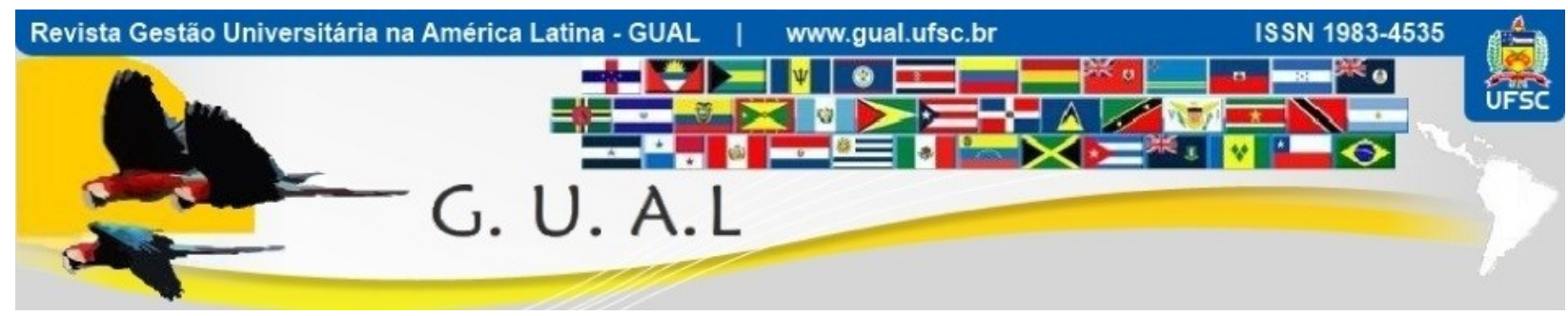

DOI: http://dx.doi.org/10.5007/1983-4535.2016v9n2p178

\title{
PERCEPCIÓN PRELIMINAR DE LOS MARCOS REGULATORIOS DEL SISTEMA UNIVERSITARIO IBEROAMERICANO: ALGUNOS AVANCES INVESTIGATIVOS
}

\section{PRELIMINARY PERCEPTION OF REGULATORY FRAMEWORKS IN IBEROAMERICAN'S UNIVERSITY SYSTEMS: SOME RESEARCH ADVANCEMENTS}

Francisco Ganga Contreras, Doutor Universidad de Los Lagos - Chile fganga@ulagos.cl

Recebido em 07/junho/2014

Aprovado em 27/novembro/2015

Sistema de Avaliação: Double Blind Review

Esta obra está sob uma Licença Creative Commons Atribuição-Uso. 


\begin{abstract}
RESUMEN
La universidad es una institución del medievo, de carácter social y altamente respetada por las respectivas comunidades que la acogen, que ha demostrado una versatilidad a toda prueba al mantenerse vigente por tantos años, no obstante, ésta debe ser capaz de expresar su naturaleza comunitaria en la capacidad de estar al servicio de la sociedad que le dio vida, y por ende, requiere estar sujeta a los marcos establecidos para asegurar su adecuado funcionamiento.

Tomando en cuenta esta realidad, se ha estimado conveniente realizar una indagación primaria cuya finalidad central es conocer la percepción que tienen los entendidos en gestión universitaria respecto al marco regulatorio en Iberoamérica. De lo que se trata definitivamente es de la presentación de avances preliminares, y para alcanzar este objetivo se procedió a la aplicación de una encuesta con preguntas cerradas a una muestra no probabilística e intencionada de 149 expertos pertenecientes a diferentes universidades iberoamericanas; siendo ocho variables que se analizan a partir de un cuestionario auto aplicado a diferentes funcionarios universitarios.
\end{abstract}

Palabras claves: Universidades. Sistema universitario. Marcos regulatorios. Teoría de agencia.

\begin{abstract}
The university is an institution of medieval origin of a social nature, and highly respected by the communities that are home, which has shown a clear versatility, while keeping its effect to over the years; but its Community nature, must be expressed specifically in their ability to serve the society that gave life and therefore should be subject to established frameworks to ensure proper operation.

Given this reality, it has been considered appropriate to perform a primary inquiry whose central purpose is to know the perception of the university management experts regarding the university regulatory framework in Latin America; It is ultimately the presentation of preliminary progress. To achieve this objective, we proceeded to the implementation of a survey with closed questions to a non-probabilistic intentional sample of 149 experts from different universities in Latin America. 8 variables which are analyzed, from a questionnaire applied to various university officials.
\end{abstract}

Keywords: Universities. University system. Regulatory frameworks. Agency theory. 


\section{INTRODUCCIÓN}

En la actualidad existe cierto consenso en reconocer que la universidad está inmersa en una multiplicidad de mega tendencias fijadas por el imperativo de actuar cada día con mayores niveles de cognición estratégica, lo cual implica -entre otras cosas- ensalzar el conocimiento y la forma de operacionalizarlo, como un factor crítico de éxito; junto con ello, debe replantearse con mirada de largo plazo (luces largas como dirían algunos o binóculos de larga vista dirían otros) su rol en una sociedad altamente cambiante y con grandes expectativas vinculadas a este tipo de instituciones.

En Iberoamérica, se están analizando fórmulas para asegurar la eficiencia en la administración de las universidades. En este sentido, muchos concuerdan en que la gestión del gobierno universitario es uno de los factores determinantes para perfeccionar el quehacer administrativo y, desde ahí, las tareas académicas.

Lo anterior es de extrema relevancia y trascendencia, si se considera -como se expresó anteriormente- que la universidad es una institución de origen medieval y de carácter social que ha demostrado una versatilidad a toda prueba al mantenerse vigente por tantos años, no obstante, ésta debe ser capaz de expresar su naturaleza comunitaria al mostrar su vocación para estar al servicio de la sociedad que le dio vida, y por ende, requiere de marcos establecidos que aseguren su adecuado funcionamiento.

Tomando en consideración estos elementos contextuales, el trabajo se ha planteado conocer la apreciación de especialistas en gestión respecto al marco regulatorio universitario existente en sus respectivos países. Se procedió a la aplicación de una encuesta con preguntas cerradas a una muestra no probabilística e intencionada de ciento cuarenta y nueve casos pertenecientes a diferentes universidades iberoamericanas. Se analizan ocho variables de un cuestionario auto aplicado a diferentes funcionarios universitarios denominados en el marco de esta investigación como "expertos".

Como hallazgos relevantes se pueden destacar la identificación y la reflexión en torno a elementos y factores determinantes considerados por la teoría de agencia, aplicables al mundo de la gestión universitaria. Asimismo, se observa generalmente una actitud dividida en relación a la calidad y la eficiencia de los diferentes sistemas de control de las

${ }^{1}$ En esta investigación se entiende por expertos a personas que ocupen o hayan ocupado cargos de gestión: jefes de área, carrera y/o programas, directores, decanos, vicerrectores y Rectores. También pueden contestarla académicos y profesionales que estudien, tengan experiencia o conozcan los temas de gobierno organizacional o sistemas universitarios. 
universidades iberoamericanas. Sin embargo, la postura crítica es levemente mayor. Lo anterior significa que podría mejorarse la legislación y normativa existente, pero también podría perfeccionarse el nivel de intervención de algunos organismos externos.

Se debe subrayar además la mirada concreta que se tiene de la legislación y los sistemas de control de las universidades: algunos creen que son los adecuados, sin embargo, otros plantean que la normativa y la legislación de sus países originan ineficiencia en la gestión o que el nivel de control externo es prácticamente inexistente.

\section{DESARROLLO}

\subsection{ASPECTOS METODOLÓGICOS}

Este trabajo se ha trazado como objetivo de carácter general conocer de manera preliminar la percepción de una muestra de expertos en gestión universitaria, sobre los marcos regulatorios de las universidades de Iberoamérica.

De este macroobjetivo se desprenden dos propósitos específicos:

- Indagar en la apreciación de expertos en gestión universitaria respecto a la legislación que regula el sistema.

- Conocer la opinión de éstos acerca de la calidad de los sistemas de control de gestión a los que están sometidas las universidades.

Para alcanzar las metas planteadas, se realiza una indagación inicial de carácter exploratorio, que proporciona un adelanto de una investigación más global, que se efectúa en el marco de un proyecto financiado con el Fondo Nacional de Desarrollo Científico y Tecnológico (FONDECYT-Regular), que lleva por título "Sistemas de Gobierno de las Universidades Iberoamericanas: Un análisis desde la Teoría de Agencia”.

En este caso particular se analizan ocho variables de un cuestionario auto aplicado a entendidos en el tema de las universidades, denominados en el marco de esta investigación como expertos. Se entiende como tales a funcionarios con experiencia en gestión desde algún cargo administrativo de relevancia o dada su participación en la docencia e investigación. Se trabaja con ciento cuarenta y nueve casos pertenecientes a diferentes entidades de Iberoamérica.

En la tabla $\mathrm{N}^{\circ} 1$ se presenta un resumen de los países y los tipos de entidades de Educación Superior a las que pertenecen los integrantes de la muestra de estudio según su institución y su nación: 
Tabla $\mathbf{N}^{\circ} 1$ Muestra de expertos utilizados en el estudio.

\begin{tabular}{|l|c|c|c|c|c|}
\hline \multicolumn{1}{|c|}{ País } & $\begin{array}{c}\mathbf{N}^{\mathbf{0}} \text { de Us. } \\
\text { Estatales }\end{array}$ & $\begin{array}{c}\mathbf{N}^{\mathbf{o}} \mathbf{d e} \text { Us. } \\
\text { Privadas }\end{array}$ & $\begin{array}{c}\mathbf{N}^{\mathbf{o}} \mathbf{d e} \text { Us. } \\
\text { Confesionales }\end{array}$ & $\begin{array}{c}\text { Otra } \\
\text { Institución } \\
\text { Educacional }\end{array}$ & Total \\
\hline Argentina & 20 & 2 & 0 & 0 & 22 \\
\hline Brasil & 19 & 5 & 2 & 0 & 26 \\
\hline Chile & 16 & 11 & 10 & 1 & 38 \\
\hline Colombia & 5 & 4 & 6 & 1 & 16 \\
\hline Costa Rica & 1 & 0 & 0 & 0 & 1 \\
\hline Ecuador & 9 & 0 & 0 & 0 & 9 \\
\hline España & 9 & 0 & 0 & 0 & 9 \\
\hline Guatemala & 1 & 0 & 0 & 0 & 1 \\
\hline México & 10 & 1 & 1 & 0 & 12 \\
\hline Paraguay & 4 & 0 & 0 & 0 & 6 \\
\hline Perú & 0 & 3 & 3 & 0 & 1 \\
\hline Uruguay & 1 & 0 & 0 & 0 & 4 \\
\hline Venezuela & 4 & 0 & 0 & $\mathbf{2}$ & $\mathbf{1 4 9}$ \\
\hline Total & $\mathbf{9 9}$ & $\mathbf{2 6}$ & $\mathbf{2 2}$ & 0 \\
\hline
\end{tabular}

Fuente: Elaboración propia, basado en encuesta aplicada a “expertos” de universidades de Iberoamérica.

\subsection{LA TEORÍA DE AGENCIA, COMO BASE TEÓRICA}

La concepción epistemológica de esta indagación parte de la premisa de que el sujeto humano, en general, tiende a actuar inspirado y motivado por sus propias aspiraciones y/o anhelos alejándose de los propósitos de la organización en su conjunto. De ahí que se requieran mecanismos que permitan encausar o limitar el actuar de las personas hacia lo que espera de ellos su organización y de ahí precisamente surgen los marcos regulatorios. Una de las bases teóricas que aborda de mejor manera tal postura es la teoría de agencia, conocida también como "enfoque principal-agente".

Esta teoría se basa en los postulados de Jensen y Mecling (1976), los que sostienen que en todo gobierno de las organizaciones existen dos actores: agente y principal (de ahí una de sus nominaciones). Entre ellos se celebran contratos (tácitos y/o explícitos), donde el mandatario (agente) es contratado por un mandante (principal), para que realice un determinado servicio en su nombre, lo cual implica cierto grado de delegación de autoridad en el agente.

Complementando la anterior definición, Gorbaneff (2003), sostiene que cuando se delega autoridad de decisión en el agente surgen cuatro problemas a saber:

a)La información entre principal y agente es asimétrica,

b)La acción del agente no es directamente observable por el principal,

c)El resultado de las acciones del agente no depende sólo de él, sino también de los choques externos, 
d)El principal y el agente son racionales y buscan maximizar sus funciones de utilidad, que no coinciden.

En correspondencia con la información asimétrica, uno de los problemas vertebrales que identifica la teoría de agencia, Bueno y Santos (2012), apuntan a que esta situación se expresa como: selección adversa, efecto señalización y riesgo moral (estos tres elementos que perfeccionan el planteamiento de Gorbaneff, se explican en la figura $\mathrm{N}^{\circ} 1$ ).

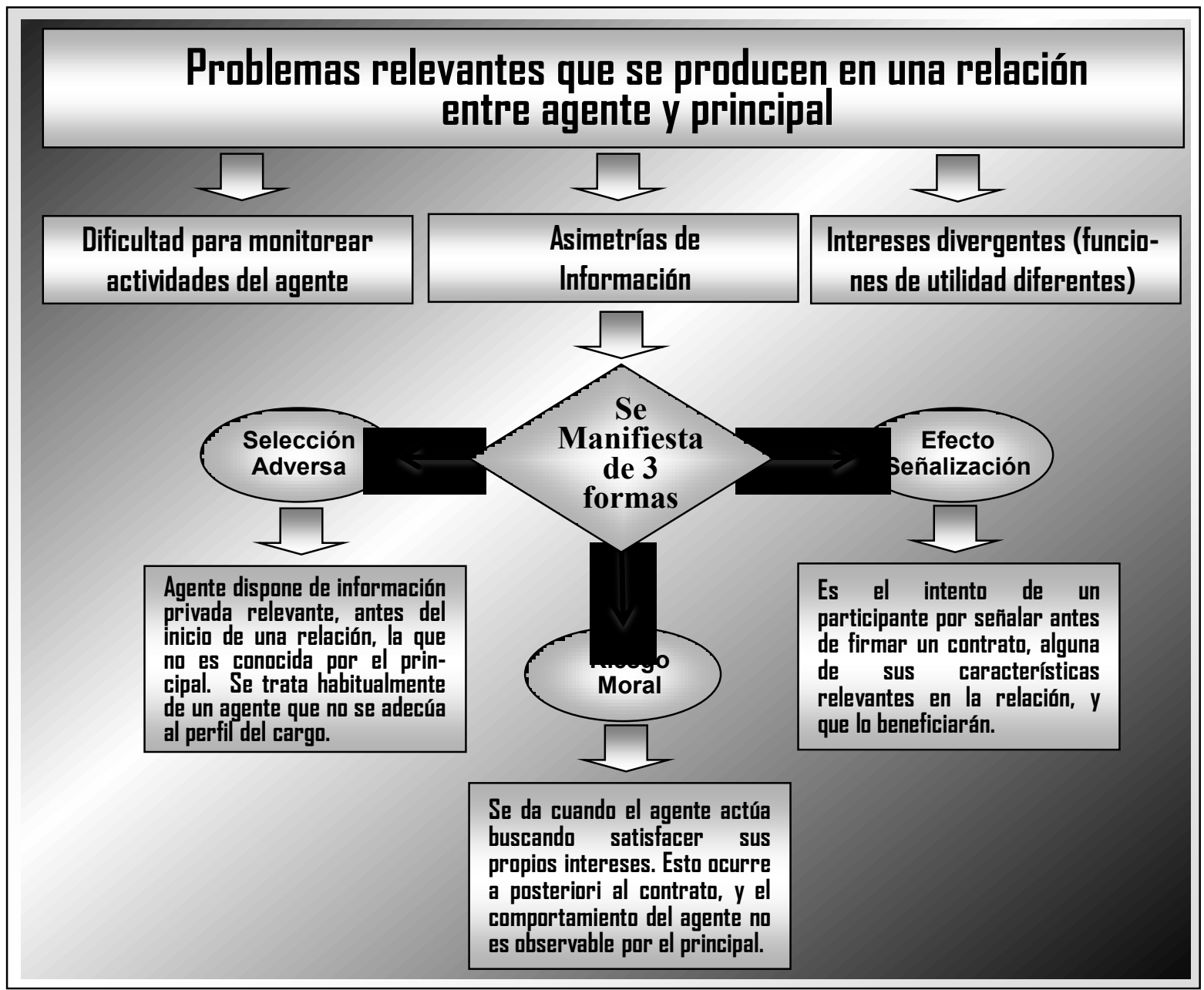

Figura $\mathbf{N}^{\circ} 1$ Dificultades en la relación de agencia y expresiones concretas de la asimetría de información. Fuente: Elaboración propia, basado en Gorbaneff 2003, Montoya y Montoya 2005, Bueno y Santos 2012, PerisOrtiz et al 2012, Ganga et al 2014.

En este breve escenario teórico se manifiesta concretamente que es muy importante tomar conciencia de la relevancia de ciertos dispositivos explícitos que ayudan a resolver los problemas que se detectan habitualmente en el enfoque "Principal-Agente" (información asimétrica, dificultada para observar actividades del agente e intereses divergentes). Aparece como un elemento central la mirada que se pueda tener de los marcos regulatorios, y la forma 
como éstos consideran y fortalecen el tipo de control al cual está sometida la institución, por ejemplo.

\subsection{PRESENTACIÓN DE RESULTADOS}

Lo primero que se busca indagar tiene que ver con el tipo de control más recurrente al que se someten las universidades iberoamericanas: se detectó que prácticamente el 37\% de los expertos, que conforman el grupo estudiado, opinaron que el control de gestión más común en sus organizaciones es el "de procesos" 2 en cambio, un poco más del $25 \%$ manifiestó que el más habitual es el "dé por resultados"; y cerca de un tercio de ellos, el $(30,9 \%)$ planteó que “ambos tipos" (de procesos y por resultados) son habituales en la supervisión de la gestión en sus instituciones, tal como se describe los en la figura $\mathrm{N}^{\circ} 2$ :

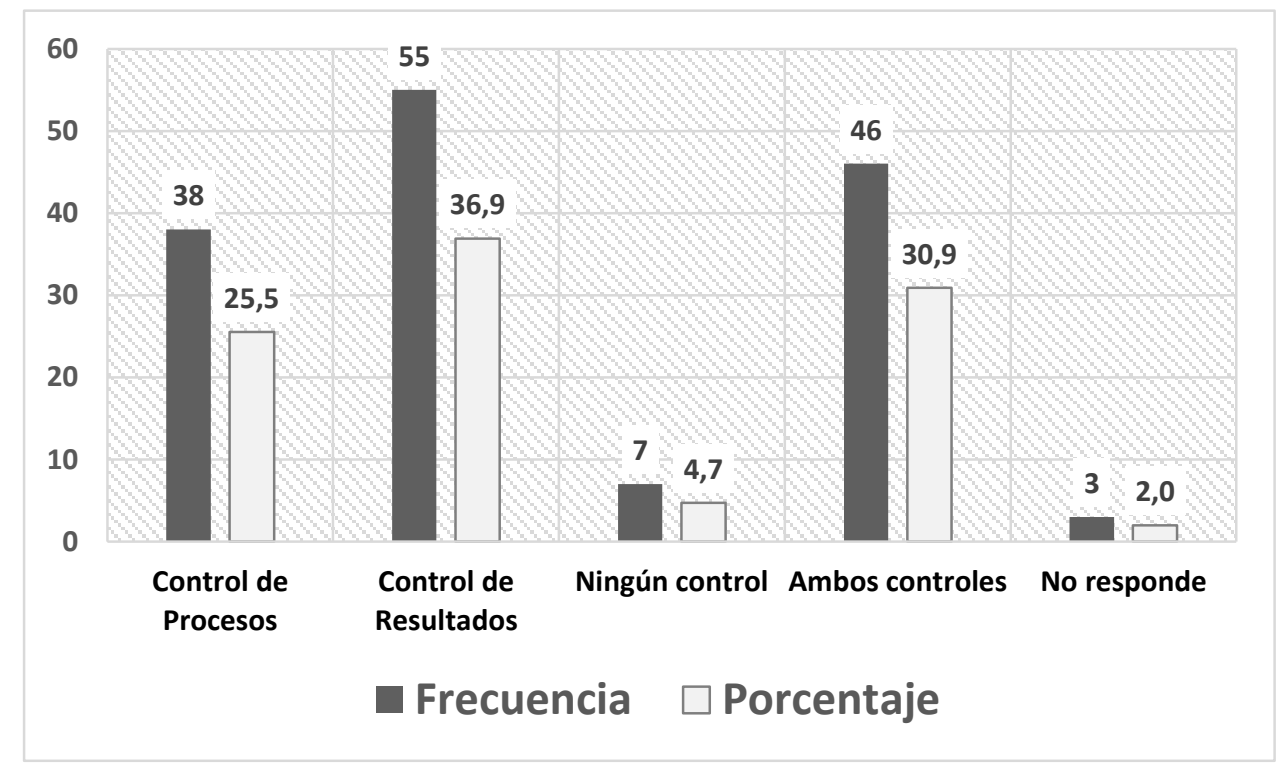

Figura $\mathbf{N}^{\circ} 2$ Tipo de control más habitual a la que se somete la gestión de su universidad. Fuente: Elaboración propia, basado en encuesta aplicada a "expertos" de universidades de Iberoamérica.

Cuando se solicitó a los expertos que evaluaran el sistema de control externo de sus casas de estudios, más de la mitad expresó que los de sus universidades son "buenos"; alrededor del 5\% los catalogó como "muy buenos", y más de un tercio opinó que es "malo" y "muy malo" (ver tabla $\mathrm{N}^{\circ} 2$ ).

${ }^{2}$ Se entiende como control de procesos a la supervisión de su comportamiento de forma habitual, mientras que el por resultados se refiere a un control por cumplimiento de objetivos en un período pre fijado. 
Tabla $\mathbf{N}^{\circ} 2$ Evaluación del sistema de control interno.

\begin{tabular}{|l|c|c|}
\hline & Frecuencia & Porcentaje \\
\hline Muy bueno & 7 & $4,7 \%$ \\
\hline Bueno & 81 & $54,4 \%$ \\
\hline Malo & 51 & $34,2 \%$ \\
\hline Muy malo & 3 & $2,0 \%$ \\
\hline No responde & 7 & $4,7 \%$ \\
\hline Total & $\mathbf{1 4 9}$ & $\mathbf{1 0 0} \%$ \\
\hline
\end{tabular}

Fuente: Elaboración propia, basado en encuesta aplicada a "expertos" de universidades de Iberoamérica.

Como puede apreciarse en la tabla $\mathrm{N}^{\circ} 3$, en cuanto al sistema de control externo, un alto porcentaje de los encuestados (más del 60\%) opinan que es "bueno" o "muy bueno" y como contrapartida, un poco más del $30 \%$ cree que es un sistema "malo" o "muy malo".

Tabla No 3 Evaluación el sistema de control externo.

\begin{tabular}{|l|c|c|}
\hline & Frecuencia & Porcentaje \\
\hline Muy bueno & 7 & $4,7 \%$ \\
\hline Bueno & 83 & $55,7 \%$ \\
\hline Malo & 43 & $28,9 \%$ \\
\hline Muy malo & 2 & $1,3 \%$ \\
\hline No responde & 14 & $9,4 \%$ \\
\hline Total & $\mathbf{1 4 9}$ & $\mathbf{1 0 0} \%$ \\
\hline
\end{tabular}

Fuente: Elaboración propia, basado en encuesta aplicada a “expertos” de universidades de Iberoamérica.

De igual manera fue objeto de exploración la mirada de los expertos en relación a la normativa y la legislación de cada país. Las respuestas arrojaron opiniones divididas. En este sentido, cerca de la mitad de los encuestados declaró que la normativa y las leyes de su nación son "buenas" o "muy buenas", el porcentaje de aquellos que opinaron que son "malas" o "muy malas" supera levemente el 50\%, como se pueden ver a continuación en la figura $\mathrm{N}^{\circ} 3$. 


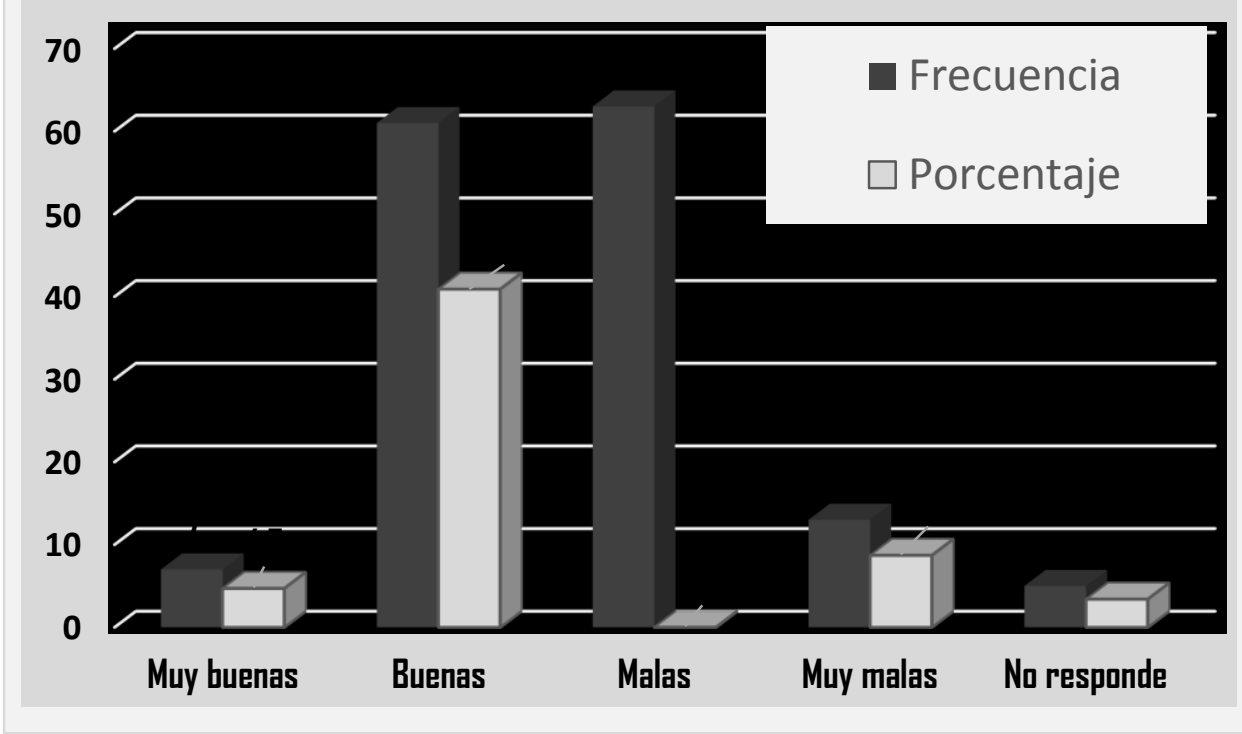

Figura $\mathbf{N}^{\circ} 3$ Evaluación de las actuales normativas y leyes en materia de Educación Superioren su país. Fuente: Elaboración propia, basado en encuesta aplicada a "expertos" de universidades de Iberoamérica.

Si bien los discernimientos en cuanto a la normativa y la legislación están fraccionados, la mayor parte de las respuestas - cerca del 60\% de ellas- dan cuenta de que la legislación ha afectado la eficiencia en la gestión de las universidades donde trabajan los encuestados, y sólo una cifra superior al tercio de éstos piensa que la legislación no afecta la eficiencia de la gestión universitaria; es llamativo también el hecho de que casi el $10 \%$, no sabe o no desea contestar esta pregunta (ver tabla $\left.\mathrm{N}^{\circ} 4\right)$.

Tabla $\mathbf{N}^{\circ} 4$ ¿Cree usted que la legislación actual ha afectado la eficiencia de la gestión de la(s) universidad (es) donde trabaja?

\begin{tabular}{|l|c|c|}
\hline & Frecuencia & Porcentaje \\
\hline $\mathrm{Si}$ & 89 & $59,7 \%$ \\
\hline No & 46 & $30,9 \%$ \\
\hline No sabe & 12 & $8,1 \%$ \\
\hline No responde & 2 & $1,3 \%$ \\
\hline Total & $\mathbf{1 4 9}$ & $\mathbf{1 0 0} \%$ \\
\hline
\end{tabular}

Fuente: Elaboración propia, basado en encuesta aplicada a “expertos” de universidades de Iberoamérica.

Este estudio preliminar mostró, complementariamente, que más del $36 \%$ de quienes respondieron manifestó estar "en desacuerdo" con la afirmación de que la normativa que regula las universidades no permite a los máximos cuerpos colegiados monitorear adecuadamente la labor del rector, no obstante, existe un poco más del $30 \%$ que concuerda 
con dicha aseveración, mientras que un porcentaje similar no está "de acuerdo ni en desacuerdo" (Ver Tabla $\mathrm{N}^{\circ}$ 5).

Tabla $\mathbf{N}^{\circ} 5$ Las normativas que regulan el funcionamiento de las universidades en mi país, no permiten a los máximos cuerpos colegiados monitorear adecuadamente la labor del rector.

\begin{tabular}{|l|c|c|}
\hline & Frecuencia & Porcentaje \\
\hline Totalmente en desacuerdo & 16 & $10,7 \%$ \\
\hline En desacuerdo & 39 & $26,2 \%$ \\
\hline Ni en desacuerdo, ni de acuerdo & 45 & $30,2 \%$ \\
\hline De acuerdo & 33 & $22,1 \%$ \\
\hline Totalmente de acuerdo & 14 & $9,4 \%$ \\
\hline No responde & 2 & $1,3 \%$ \\
\hline Total & $\mathbf{1 4 9}$ & $\mathbf{1 0 0 \%}$ \\
\hline
\end{tabular}

Fuente: Elaboración propia, basado en encuesta aplicada a “expertos” de universidades de Iberoamérica.

Finalmente, se intenta conocer la opinión de los expertos en relación a la eficiencia que perciben de los sistemas de control, en sus respectivos países. Al respecto, se detectó que más del $46 \%$ piensa que son ineficientes; en el lado contrario, el porcentaje de respuestas supera levemente el $30 \%$. En la figura $\mathrm{N}^{\circ} 4$, se pueden observar mayores detalles de los expresado previamente.

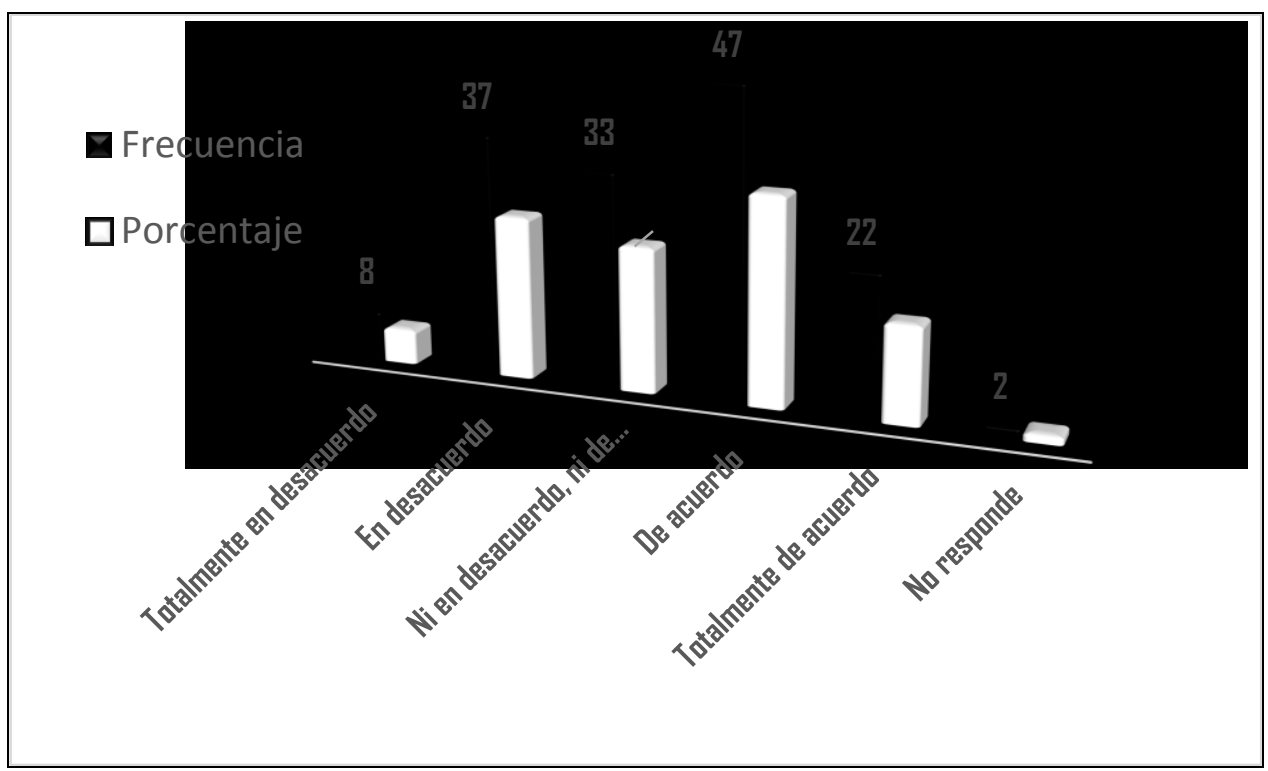

Figura $\mathbf{N}^{\circ} 4$ Los sistemas de control de las universidades en mi país son ineficientes.

Fuente: Elaboración propia, basado en encuesta aplicada a "expertos" de universidades de Iberoamérica.

En forma más específica y desde otra perspectiva se preguntó sobre cuáles son los organismos externos que supervisan la gestión de las universidades, y en qué nivel ejercen 
éste control. En la Tabla $N^{\circ} 6$ se exponen las entidades que ejercen control externo a la gestión según el país al que pertenecen y el nivel de control promedio ${ }^{3}$

El Ministerio de Educación constituye una de las estructuras reguladoras más mencionadas, aunque no representa necesariamente el organismo que ejerce un mayor control. De igual modo, muchos de los encuestados mencionan instituciones públicas como las contralorías, cuyo deber es supervisar a la universidad dada la utilización de fondos públicos en su ejecución.

Por otro lado, fue posible comprobar que en varios de los países existen entidades dedicadas exclusivamente a fiscalizar a las universidades, las que se encargan de la acreditación de las mismas y/o buscando asegurar o reconocer la calidad, verbi gratia: ANUIES (Asociación Nacional de Universidades e Instituciones de Educación Superior), en México; CONEAU (Comisión Nacional de Evaluación y Acreditación Universitaria) en Argentina; CNA (Comisión Nacional de Acreditación) en Chile; CAPES (Coordinación de Perfeccionamiento de Personas de Nivel Superior) en Brasil; SINAES (Sistema Nacional de Acreditación de la Educación Superior) EN Costa Rica o el CEAACES (Consejo de Evaluación, Acreditación, y Aseguramiento de la Calidad de la Educación Superior) en Ecuador.

En lo relativo a los niveles del control que ejerce cada organismo externo se observan promedios disímiles: Brasil y Ecuador caen en el rango de suficiente control a mucho control; Chile, Argentina, España y México varían entre poco control y a un control suficiente.

\footnotetext{
${ }^{3}$ El nivel de control se mide en una escala de 1 a 5 , donde 1 significa "ningún control” y 5 "mucho control".
} 
Tabla $N^{\circ} 6$ Organismo de control externo según país.

\begin{tabular}{|c|c|c|c|}
\hline País & $\underline{\text { Organismo de control }}$ & $\begin{array}{l}\text { Número de } \\
\text { Menciones }\end{array}$ & $\begin{array}{c}\text { Nivel de } \\
\text { control } \\
\text { promedio }\end{array}$ \\
\hline \multirow{3}{*}{ Argentina } & Ministerio de Educación de la Nación Argentina & 9 & 3,11 \\
\hline & $\begin{array}{l}\text { Comisión Nacional de Evaluación y Acreditación Universitaria, } \\
\text { CONEAU }\end{array}$ & 6 & 4 \\
\hline & Sindicatura General de la Nación, SIGEN & 5 & 3,4 \\
\hline \multirow{3}{*}{ Brasil } & Ministerio de Educación y Cultura & 19 & 4,05 \\
\hline & $\begin{array}{l}\text { Coordinación de Perfeccionamiento de Personas de Nivel } \\
\text { Superior, CAPES (Control de Calidad de Post Grado) }\end{array}$ & 9 & 4 \\
\hline & Tribunal de Cuentas de la Unión & 5 & 4,6 \\
\hline \multirow{3}{*}{ Chile } & Ministerio de Educación, MINEDUC & 23 & 3,04 \\
\hline & Comisión Nacional de Acreditación, CNA & 10 & 3,6 \\
\hline & Contraloría General de la República & 7 & 4,43 \\
\hline \multirow{3}{*}{ Colombia } & Ministerio de Educación Nacional de Colombia & 12 & 3,33 \\
\hline & Contraloría & 4 & 4 \\
\hline & $\begin{array}{l}\text { Departamento Administrativo de Ciencias, Tecnología e } \\
\text { Innovación, Colciencias }\end{array}$ & 3 & 4 \\
\hline \multirow{2}{*}{ Cota Rica } & Auditoría & 1 & 3 \\
\hline & Contraloría General de la República & 1 & 5 \\
\hline \multirow{3}{*}{ Ecuador } & $\begin{array}{l}\text { Secretaría Nacional de Educación Ciencia y Tecnología, } \\
\text { SENESCYT }\end{array}$ & 8 & 4,12 \\
\hline & $\begin{array}{l}\text { Consejo de Evaluación, Acreditación, y Aseguramiento de la } \\
\text { Calidad de la Educación Superior, CEAACES }\end{array}$ & 8 & 4,88 \\
\hline & Consejo de Educación Superior, CES & 7 & 4,71 \\
\hline \multirow{3}{*}{ España } & Ministerio de Educación Cultura y Deporte & 2 & 4 \\
\hline & Cámara de Cuentas & 2 & 3 \\
\hline & Auditoría Externa & 2 & 3 \\
\hline Guatemala & Contraloría General de Cuentas & 1 & 3 \\
\hline \multirow{3}{*}{ México } & Secretaría de Educación Pública, SEP & 10 & 2,9 \\
\hline & Organismos de acreditación & 3 & 3,67 \\
\hline & $\begin{array}{l}\text { Asociación para la Acreditación y Certificación en Ciencias } \\
\text { Sociales, ACCECISO }\end{array}$ & 2 & 4,5 \\
\hline \multirow{3}{*}{ Paraguay } & Contraloría General de la República & 2 & 5 \\
\hline & Consejo Nacional de Educación & 1 & 3 \\
\hline & $\begin{array}{l}\text { Ministerio de Educación - ANEAES (Agencia Nacional de } \\
\text { Evaluación y acreditación de la Educación Superior) }\end{array}$ & 1 & 3 \\
\hline \multirow{3}{*}{ Perú } & Asamblea Nacional de Rectores (ANR) & & \\
\hline & Ministerio de Trabajo & 2 & 3,5 \\
\hline & Asociation to Advance Collegiate School of Bussines, AACSB & 2 & 3,5 \\
\hline \multirow{3}{*}{ Venezuela } & Ministerio del Poder Popular para la Educación Universitaria & & \\
\hline & Sindicatura General de la Nación & 1 & 4 \\
\hline & Contraloría General de la República & 1 & 3 \\
\hline
\end{tabular}

Fuente: Elaboración propia, basado en encuesta aplicada a “expertos” de universidades de Iberoamérica.

\section{CONCLUSIONES}

El XIV Coloquio Internacional de Gestión Universitaria en América del Sur, realizado en Brasil entre el 3 y el 5 de diciembre del 2014, permitió la presentación preliminar de algunos resultados que están en el marco de un proyecto de investigación -financiado por el 
gobierno de Chile- que concluirá en marzo del 2015. Los productos dados a conocer, como una especie de avance sinóptico, se enriquecerán -no sólo con las retroalimentaciones recibidas en el citado evento- sino también, con los comentarios que puedan emerger a partir de los resultados que recoge este documento.

Ahora bien, en general se observa en la opinión de los encuestados, un fraccionamiento respecto a la calidad y la eficiencia de los diferentes sistemas de control de las universidades iberoamericanas, sin embargo, la postura crítica es levemente mayor. En este sentido, podría desprenderse que se aspira a una mejora en la legislación y normativa existente, así como también el nivel de control de algunos organismos externos.

De igual manera afloran ciertas ideas contrapuestas dignas de destacar; mientras algunos creen que la legislación y los sistemas de control de las universidades son los adecuados, otros consideran que la normativa y legislación de sus respectivos países generan ineficiencia en la gestión, o que el nivel de control externo es inexistente.

Específicamente en lo concerniente a la legislación y normativa de cada nación la muestra de estudio arrojó división: unos la catalogan como buena y otros deficiente. Es levemente superior el porcentaje de aquellos que piensan que la legislación y normativa de la universidades es mala $(45,6 \% \mathrm{v} / \mathrm{s} 50,1 \%$, respectivamente). Asimismo, es mayor la proporción de los que estiman que la normativa y la legislación del país afectan la eficiencia de las universidades.

En lo tocante a la evaluación que se realiza de los sistemas de control a la gestión universitaria la mayoría revela estar conforme tanto con el sistema de control interno, como con el sistema de control externo. Cerca del $60 \%$ de los expertos plantean que los sistemas de control interno son buenos versus un poco más del tercio que los califica como malos. Esta proporción es muy similar a la percepción de calidad de los sistemas de control externo, donde un $60 \%$ los evalúa de buenos y sobre el $30 \%$ de malos. Ante la interrogante de qué si los sistemas de control de gestión de las universidades de su país son ineficientes, cerca de la mitad responde afirmativamente y un porcentaje levemente superior al 30\% de manera negativa. Por otra parte, es mayor el porcentaje de quienes creen que los sistemas de control son buenos, pero también es mayor el porcentaje de aquellos que lo consideran ineficientes.

Adicionalmente, es posible constatar que los países varían de acuerdo a los organismos externos que cumplen la función de supervisar a las universidades. Mientras que algunos cuentan con los organismos más genéricos de control, como las contralorías o los 
ministerios, otros cuentan con diferentes organismos especializados en acreditación y evaluación de calidad.

Entre los organismos de control externo mencionados por los encuestados, existen algunos que son calificados con un nivel de control suficiente, mientras que otros no ejercen el control suficiente de acuerdo a los expertos encuestados.

Los productos presentados aquí, solo constituyen una aproximación preliminar de los resultados consolidados que arrojarán las opiniones de más de 600 expertos en gestión universitaria de Iberoamérica, que se pretenden lograr durante los meses del 2015. Las rutas investigativas serán múltiples y amplias, lo que permitirá generar conocimiento al servicio del perfeccionamiento de los sistemas de gobierno de nuestras universidades. De igual forma, se pretende que las acotaciones que puedan emerger a partir de los resultados que proyecta el presente estudio, incidan en futuras investigaciones sobre el tema.

\section{BIBLIOGRAFÍA}

Bueno, J. y D. Santos. (2012): Teoría de la agencia en la determinación de la estructura de capital. Casos sectores económicos del departamento del Valle del Cauca. Revista Prolegómenos - Derechos y Valores. Vol. XV. N 30.

Ganga F. et al (2014): Apreciación de rectores y miembros de los máximos cuerpos colegiados de las universidades chilenas, respecto del mercado de los directivos de la Educación Superior. Revista Gaceta Laboral. Vol. 20. N 1.

Gorbaneff, Y. (2003): Teoría del agente-principal y el mercadeo. Colombia: Revista Universidad EAFIT. $\mathrm{N}^{\circ} 129$.

Jensen, M. y W. Meckling (1976): Theory of the Firm: Managerial Behavior, Agency Costs and Ownership Structure. Journal of Financial Economics. October, 1976, V. 3, No 4, pp. 305-360.

Montoya, I. y L. Montoya. (2005). Concepciones básicas en economía de la información y la selección adversa en el objeto de estudio de la teoría organizacional. Revista Economía y Desarrollo. Vol. 4. $\mathrm{N}^{\circ} 1$.

Periz-Ortiz, et al (2012): Fundamentos de la teoría organizativa de agencia. Revista Nuevas Corrientes de Pensamiento Económico. № 865. 